\title{
Enhancement of Space Plasma Images by Complex Wavelets
}

\author{
Vitor Moura Souza ${ }^{1} \cdot$ Margarete Oliveira Domingues $^{1}$ - Odim Mendes ${ }^{1}$. \\ Aylton Pagamisse $^{2}$ - Guillermo Adrian Stenborg ${ }^{3}$
}

Received: 6 March 2015 / Published online: 12 August 2015

(C) Sociedade Brasileira de Física 2015

\begin{abstract}
The Sun is a natural laboratory for plasma processes. A myriad of instruments aboard satellites and on ground record(ed) the plasma emission in different ranges of the electromagnetic spectrum to help understand such processes. In particular, in the outer part of the solar atmosphere, the solar corona, we can observe a multitude of electrodynamical phenomena. There, the faint corona emission and the associated dynamic plasma structures (e.g., coronal mass ejections-CMEs) recorded in whitelight images can be used as basis for some insight of this physical scenario. In order to characterize the dynamics and morphology of such structures in a better way, it seems crucial that some features of those images should be enhanced. To deal with this need, a new approach using a complex wavelet transform methodology was developed. With the proposed methodology, we can highlight the plasma ejections improving the identification of those structures.
\end{abstract}

Keywords Solar corona $\cdot$ Coronal mass ejections (CMEs) $\cdot$ Complex wavelet transform

Vitor Moura Souza

vitor.souza@inpe.br

1 Instituto Nacional de Pesquisas Espaciais/INPE, São José dos Campos, São Paulo, Brasil

2 Faculdade de Ciências e Tecnologia, UNESP, Presidente Prudente, São Paulo, Brasil

3 College of Science, George Mason University, Fairfax, VA, USA

\section{Introduction}

The Sun has been constantly monitored primarily due to its potential harmful effect on Earth. Coronal mass ejections [11, CMEs], magnetic clouds [3, 4] and corotating interaction regions (CIRs) are examples of the main features rising from the complex solar dynamics that may cause geomagnetic disturbances and also affect the space technologies on which our society is dependent [10]. Among other experiments designed for Sun's monitoring (e.g., STEREO,$\left.{ }^{1} \mathrm{SDO}^{2}\right)$, the large angle and spectrometric coronagraph (LASCO) on board of the Solar Heliospheric Observatory $(\mathrm{SOHO})$ spacecraft has greatly collaborated with the remote sensing of the Sun and the magnetized plasma-made structures ejected from it.

LASCO [2] comprises three coronagraphs, LASCO/C1, $\mathrm{C} 2$, and $\mathrm{C} 3$ that provide pictures of the solar corona from near the solar surface through $32 R_{\odot}\left(R_{\odot}\right.$ Sun radius). These data are particularly well suited to study the kinematic properties of CMEs such as position, velocity, and acceleration [13].

Although the LASCO experiment has provided unique observations of CMEs evolution, the LASCO raw images include the contributions from the K- and F-corona, instrumental noise, and stray light. Therefore, the visualization of the plasma structures of interest (i.e., K-coronal structures) is contingent on the removal of the background contribution.

Some efforts have been attempted in order to improve plasma structures visualization of LASCO images, mainly on CMEs. The most popular and simple method is the time differencing, or running difference images. In this

\footnotetext{
${ }^{1}$ http://stereo.gsfc.nasa.gov/

${ }^{2}$ http://sdo.gsfc.nasa.gov/
} 
method, one can see bright regions corresponding to features observed at time $t=t_{1}$, while the dark regions correspond to the features observed at time $t=t_{0}$, both at the same frame (see, e.g., [9]). One drawback of this technique is that sometimes the mixture of features in the same frame becomes too difficult to disentangle, which may lead to ambiguous interpretations of the studied phenomena.

Other modern techniques supported on the so-called multiscale analysis, in particular the wavelet transforms, have largely been used to enhance the appearance of CMEs and its internal structures on coronagraph observations [5, 7, 22-24]. As discussed in [22], solar images present hierarchical structures. From the analysis of different scale sizes, a myriad of new plasma structures may be found. Thus, choosing image-processing techniques based on multiscale properties such as wavelet transforms becomes a natural choice. One benefit of this kind of technique is that only independent images are analyzed without any need for time differencing. However, as pointed out by [24], diffraction, interference patterns, and defects of the optics like dust particles and/or imperfection of the lenses are magnified together with the structures that we are seeking to highlight. Nevertheless, in the preliminary analysis carried out here, these side effects can be neglected.

In the aforementioned works, two common characteristics can be found: (1) the usage of pre-processing techniques to deal with the unwanted background effects quoted earlier and (2) real wavelet transforms are used for image processing. As a novelty, we use in this work a two-dimensional (2D) version of a complex, quasiorthogonal, wavelet transform known as dual-tree $[15,21]$ to better visualize CMEs overall structures in LASCO/C2 field-of-view. Among other characteristics, the dual-tree possesses the property of directional selectivity which has been shown very useful in our search for the highlight of sharp edges and distinct contours of plasma structures on LASCO/C2 images once it allows one to analyze features in the image at six well-defined orientations. On the other hand, the often used Daubechies's biorthogonal wavelet transforms (DWT, e.g., [5]) can efficiently analyze only horizontal and vertical features once there is a mixture between the diagonal orientations (see [21] for more details).

Here, the images are processed without any preprocessing technique. All of them did not have a background removal. Even with this limitation, the achieved results are promising. We do not intend in this work to describe the physics underlying the events represented in the images shown here. Our proposal presents the potential of a modern and somewhat recent wavelet tool that may further help solar physicists to unambiguously interpret the physical phenomena depicted in solar images.

This paper is organized as follows: Section 2 describes the dataset used in this work. In Section 3, the complex wavelet transform is presented, along with the approach used for processing the images. After that, Section 4 shows the results and discussions, and conclusions are given in Section 5.

\section{Dataset}

The Solar Heliospheric Observatory (SOHO) is a spacecraft that orbits the First Lagrangian Point L1, at a distance towards the Sun of about 1.5 million kilometers away from the Earth. Aboard SOHO, there are many instruments designed to study the Sun from its deep core to the outer corona and the solar wind. Among them is the large angle and spectrometric coronagraph (LASCO), which produces images of the corona in the visible spectrum. A detailed description of this instrument is found in [2].

LASCO comprises three coronagraphs, $\mathrm{C} 1, \mathrm{C} 2$, and $\mathrm{C} 3$ (C1 internally occulted and $\mathrm{C} 2$ \& $\mathrm{C} 3$ externally occulted coronagraphs) that together image all plasma phenomena taking place at a distance off the Sun's center ranging from 1.1 to $32 R_{\odot}$. LASCO/C2 telescope covers a distance from 1.5 to $6 R_{\odot}$ and its pixel size is 11.4 arcsec. The level 0.5 raw images used in this work were provided by this telescope and related to the following events:

Event 1 (E1): Eruptive prominence on June 02, 1998 at 13:31 UT;

Event 2 (E2): CME on November 23, 2000 at 21:30 UT;

Event 3 (E3): CME on October 06, 2001 at 08:06 UT;

Event 4 (E4): CME on August 13, 2002 at 10:54 UT.

Level 0.5 images are raw images that have been rotated to put the solar north at the top of the image without corrections for instrument response, stray light, etc.

Each chosen event evolves mainly in a quadrant in LASCO/C2 field-of-view. E1-E4 in South-West, SouthEast, North-West, and North-East quadrants, respectively. We seek, in this way, to test our wavelet technique ability of detecting the main directional features present in those images. It is worthwhile to note that when analyzing solar images, the observer is considered to lie on the Sun surface. Therefore, the right (left) part of the image corresponds to West (East).

An occulter disk together with a white annulus to indicate Sun's surface are added in all images. They have a full 
resolution of $1024 \times 1024$ pixels and are available in FITS format.

\section{Methodology}

The so-called discrete wavelet transforms (DWT) are commonly used in image compression and extraction of information from time series and images. One possible way to perform multiscale image decomposition by means of the DWT is by using a tree of low-pass and high-pass filters [8, $12,18]$. The DWT also has found usage to highlight coronal features present on solar images (e.g., [5]).

The dual-tree complex wavelet transform (DTCWT) as designed by $[15,16]$ has been successfully applied in several areas of image processing. For more general informations concerning the DTCWT and its applications to motion estimation and compensation, denoising and deconvolution, texture analysis and synthesis, segmentation and classification, and watermaking, the reader is referred to [17]. To date, however, no work has been reported using the DTCWT for enhancing such coronal structures.

In this work, the DTCWT represents an improvement of the DWT due to its shift invariance and multidirectional selectivity in the analyzed images $[15,16]$. It generates complex wavelet coefficients by using a dual-tree of wavelet filters to obtain their real $(r)$ and imaginary (i) parts. The complex $(c)$ wavelet coefficients $d_{c, n}^{j}$ are obtained by the projection of the signal $f(x) \in L^{2}(\mathbb{R})$ onto $2^{-j / 2} \psi_{c}\left(2^{-j} x-n\right)$

$d_{c, n}^{j}=\left\langle f, 2^{-j / 2} \psi_{c}\left(2^{-j} x-n\right)\right\rangle=d_{r, n}^{j}+i d_{i, n}^{j}$,

where $i=\sqrt{-1}$ is the imaginary unit, and

$\psi_{c}(x)=\psi_{\text {tree }_{1}}(x)+i \psi_{\text {tree }_{2}}(x)$

is a complex approximately analytic wavelet, i.e., $\psi_{\text {tree }_{2}}$ is the approximate Hilbert pair of $\psi_{\text {tree }_{1}}$, denoted by $\psi_{\text {tree }_{2}} \approx$ $\mathcal{H}\left\{\psi_{\text {tree }_{1}}\right\}$.

The complex scaling functions $\phi_{c}(x)$, which act as lowpass filters, are similarly defined

$\phi_{c}(x)=\phi_{\text {tree }_{1}}(x)+i \phi_{\text {tree }_{2}}(x)$.

We explore the two-dimensional (2D) case which is wellsuited for analyzing and processing oriented singularities in images, i.e., edges [21].

Applied on an image the 2D DTCWT generates as outcome six subimages (at each scale $j$ ) constituted by complex wavelet coefficients $d_{c}^{j^{(\theta)}}$

$d_{c}^{j^{(\theta)}}=\left\langle f, \psi_{c}^{j^{(\theta)}}(x, y)\right\rangle$.
These subimages are strongly oriented at the angles $\theta=15^{\circ}, 45^{\circ}, 75^{\circ},-75^{\circ},-45^{\circ}$, and $-15^{\circ}$. Each one of these orientations is associated with either real or imaginary parts of the following six two-dimensional complex wavelets $\psi_{c}^{j^{(\theta)}}(x, y) \in L^{2}\left(\mathbb{R}^{2}\right)$

$$
\begin{aligned}
\psi_{c}^{j^{\left(15^{\circ}\right)}}(x, y) & =\psi_{c}^{j}(x) \bar{\phi}_{c}^{j}(y), \\
\psi_{c}^{j^{\left(-15^{\circ}\right)}}(x, y) & =\phi_{c}^{j}(x) \psi_{c}^{j}(y), \\
\psi_{c}^{j^{\left(45^{\circ}\right)}}(x, y) & =\psi_{c}^{j}(x) \bar{\psi}_{c}^{j}(y), \\
\psi_{c}^{j^{\left(-45^{\circ}\right)}}(x, y) & =\psi_{c}^{j}(x) \psi_{c}^{j}(y), \\
\psi_{c}^{j^{\left(75^{\circ}\right)}}(x, y) & =\phi_{c}^{j}(x) \bar{\psi}_{c}^{j}(y), \\
\psi_{c}^{j^{\left(-75^{\circ}\right)}}(x, y) & =\psi_{c}^{j}(x) \phi_{c}^{j}(y),
\end{aligned}
$$

where

$\psi_{c}^{j}(\cdot)=2^{-j / 2} \psi_{c}\left(2^{-j} \cdot-n\right)$,

$\phi_{c}^{j}(\cdot)=2^{-j / 2} \phi_{c}\left(2^{-j} \cdot-n\right)$,

and $\psi_{c}(\cdot)$ and $\phi_{c}(\cdot)$ obey (2) and (3), respectively. Here, the dot stands for either $x$ or $y$.

The website of Prof. Nick Kingsbury ${ }^{3}$ has information on how to obtain the DT CWT codes, and most of the available material on it.

Our approach is based on enhancement of the complex wavelet coefficients $d_{c}^{j^{(\theta)}}$ over the different scales $j$. This is achieved by multiplication of $d_{c}^{j^{(\theta)}}$ by a factor $\alpha_{\theta}^{j} \in \mathbb{R}$, at a scale $j$ and at an orientation $\theta$. By increasing these coefficients, the oriented singularities in the image, i.e., the edges, are highlighted. This follows from the fact that the magnitude of the wavelet coefficients is related to abrupt changes in the signal, or "details" $[8,18]$.

In this process, there might be some directions $(\theta)$ that hold more information about the plasma structure. For this reason, the corresponding wavelet coefficients undergo a higher amplification than the other ones. Therefore, we establish a way for quantifying the amount of information contained in a specific direction in order to indicate the orientation whose wavelet coefficients should be more amplified. We do this by means of the following ratio

$R=\frac{\sum_{\text {subimage }}\left|d_{c}^{j^{(\theta)}}\right|^{2}}{\sum_{\text {all subimages }}\left|d_{c}^{j^{(\theta)}}\right|^{2}}$,

where the numerator represents the sum of all squared wavelet coefficients absolute values for a given subimage (orientation) and the denominator is the sum of all squared wavelet coefficients absolute values for all subimages. This

\footnotetext{
$\overline{3^{3} \mathrm{http} / / / w w w-s i g p r o c . e n g . c a m . a c . u k / M a i n / N G K ~}$
} 
Fig. 1 Scheme showing how the 2D DTCWT outcomes (subimages) are visualized. The color-coded regions specify in which resolution level (scale) the subimages are seen: red stands for scale $j=1$; green $j=2$, and blue $j=3$. At the top center, the original image being analyzed is seen on the scale $j=3$. Subimages sizes $\left(N_{\text {sub }} \times N_{\text {sub }}\right)$ are scaled as $N_{\text {sub }}=N / 2^{j}$, where $N=1024$ is the number of pixels in one dimension of the original image size $(N \times N)$. The top center panel is seen out of scale for visualization convenience

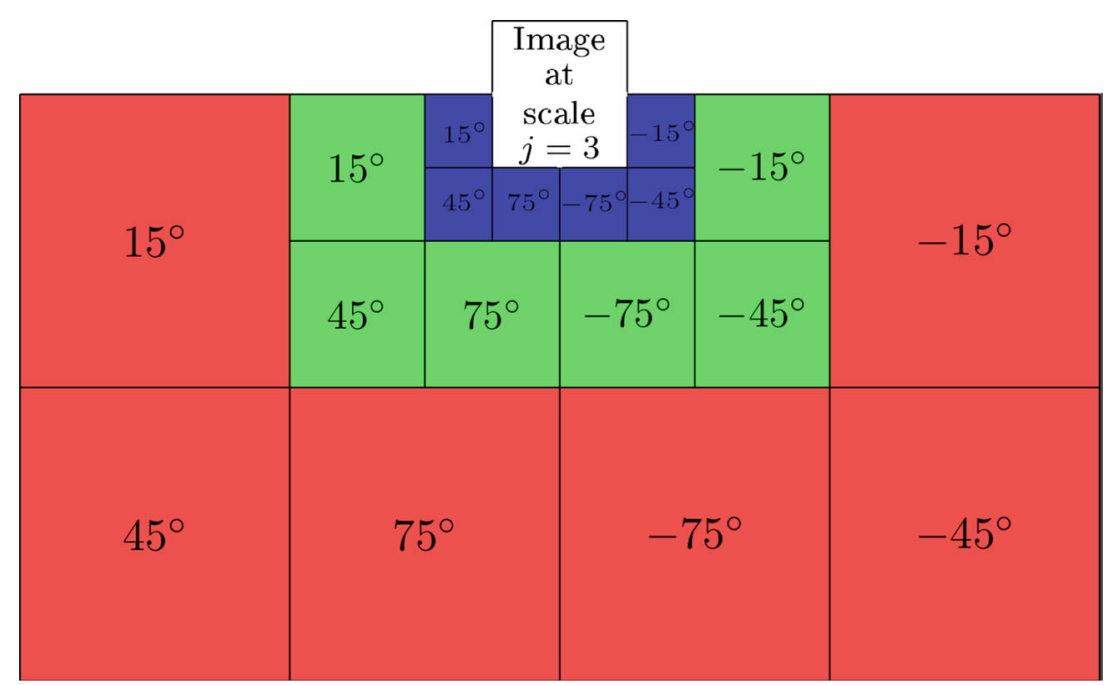

ratio is calculated for the current scale $j$. It represents a fraction of the total amount of relevant information, i.e., the "details," contained in the current scale $j$.

After successive tests, we identified that where $R<$ 0.200 the corresponding wavelet coefficients are amplified by the same factor $\alpha_{\theta}^{j}$. Otherwise, they undergo a larger amplification.

All images representing Events $E 1-E 4$ are decomposed by the 2D DTCWT into four scales $(j=1$ to 4$)$. Their outcomes (subimages) are visualized following the scheme depicted in Fig. 1. For space convenience, only three scales $j=1,2$ and 3 are shown. As a consequence of the downsampling process, i.e., reduction of the image size by half, subimages sizes, $N_{\text {sub }} \times N_{\text {sub }}$, scales as $N_{\text {sub }}=N / 2^{j}$ where $N=1024$ is the number of pixels in one dimension of the original image size $(N \times N)$. At the top center panel, the original image being analyzed is seen on the scale $j=3$. It is shown out of scale only for visualization convenience.

\section{Results and Discussion}

In this section, we discuss (i) the dual-tree's directionality property jointly with the choice of the $\alpha$ parameter in order to indicate which wavelet coefficients should undergo a higher increase prior to image's reconstruction, and afterwards (ii), the reconstructed images are shown as results from the analysis carried out in (i).

\section{Dual-Tree's Directionality Property and the Choice} of the $\boldsymbol{\alpha}$ Parameter Following the visualization scheme depicted in Fig. 1, Fig. 2 shows the outcomes from a four scale (only three are shown) 2D DTCWT applied on the image related to $E 1$. For the panels showing the subimages at the scale $j=1$, i.e., the largest panels, one is able to note that few features can be seen from the plasma structure, which is indicated at the top center panel bounded by a white rectangular box. This characteristic was found
Fig. 2 Outcomes from a three scale 2D DTCWT

decomposition performed on the image related to $E 1$. They are organized as shown in Fig. 1. The white rectangular box in the top center panel bounds the plasma structure being analyzed

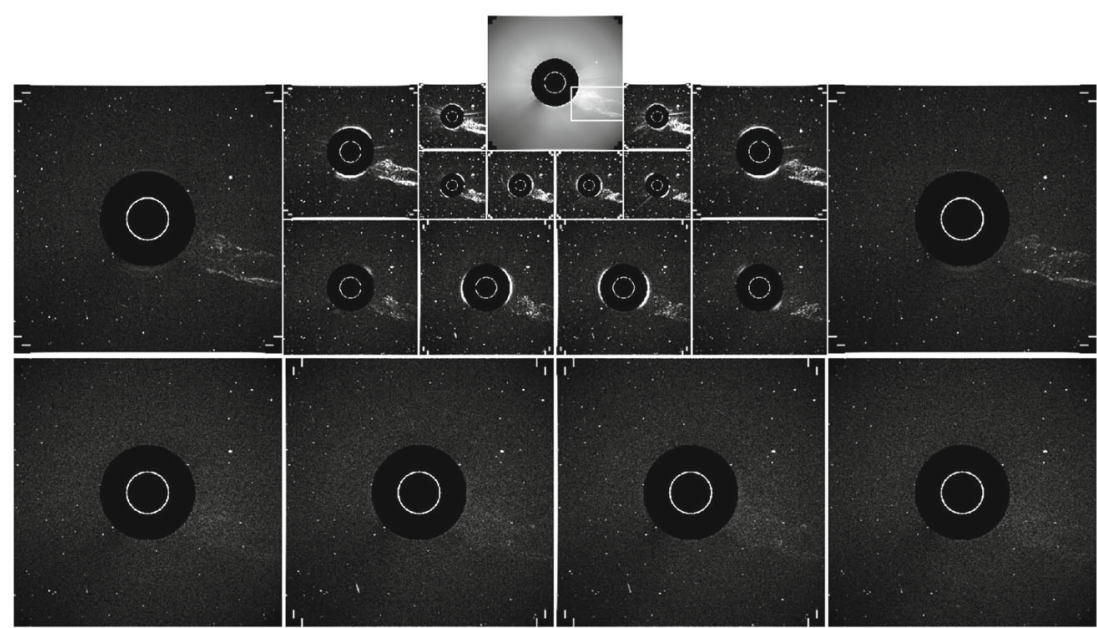


in the whole set of images analyzed in this work. Features present themselves very faint at this particular scale. This is probably associated with the large level of noise often found at the scale $j=1$. We would like to avoid noise. Then, as a first step in the image processing, we multiply all the wavelet coefficients of the subimages pertaining to this scale by a factor $\alpha_{\theta}^{j=1}$, where $0 \leq \alpha_{\theta}^{j=1}<1$. We do not use the $R$ parameter values for this particular scale to guide our analysis since we do not want to enhance the corresponding wavelet coefficients, instead we try to minimize the noise effect by reducing their absolute values. It is noticeable in Fig. 2 for the subimages at scales $j=2$ and 3 , the plasma structure becomes more highlighted, mainly at $\theta= \pm 15^{\circ}$ orientations. This visual tendency is maintained even for the subimages at scale $j=4$ (not shown). This is a qualitative indicator that the wavelet coefficients associated with these scales and orientations contain more information related to the plasma structure, whose visualization we are seeking to enhance. Therefore, we adopted that, in general, the coefficients at scale $j=3$ undergo a higher increase than those at scale $j=2$. Scale $j=$ 4 coefficients, however, did not undergo an enhancement greater than or equal to scale $j=3$ coefficients. Instead, they underwent a multiplication by a factor $\alpha_{\theta}^{j=4}$ so that, for $E 1,1<\alpha_{\theta}^{j=4}<\alpha_{\theta}^{j=2}$. In this way, the reconstructed images were better visualized. The $\alpha_{\theta}^{j}$ values were chosen in such a way that the reconstructed images provided a good contrast between the plasma structure and the background.

In order to proceed with the wavelet coefficients enhancement, it is necessary to check, for a specific scale, whether the orientations $\theta= \pm 15^{\circ}$ being discussed in the analyzed case $(E 1)$ indeed hold more information, i.e., larger $R$ values, about the plasma structure. Table 1 shows both $R$ and $\alpha_{\theta}^{j}$ values for $E 1-E 4$. By inspection of $E 1$ (top left) table, one can see that $R$ values are indeed larger for $\theta= \pm 15^{\circ}$ orientations on both scales $j=2\left(R_{+15^{\circ}}=\right.$ $\left.0.466, R_{-15^{\circ}}=0.480\right)$ and $j=3\left(R_{+15^{\circ}}=0.417\right.$, $\left.R_{-15^{\circ}}=0.415\right)$. Furthermore, their values are greater than the established threshold $(R \geq 0.200)$ for the $R$ parameter. Therefore, the corresponding wavelet coefficients will undergo a higher enhancement factor, as shown in Table 1 top right table: $\alpha_{ \pm 15^{\circ}}^{j=2}>\alpha_{\left\{ \pm 45^{\circ}, \pm 75^{\circ}\right\}}^{j=2}$ and $\alpha_{ \pm 15^{\circ}}^{j=3}>$ $\alpha_{\left\{ \pm 45^{\circ}, \pm 75^{\circ}\right\}}^{j=3}$.

It is important to notice that although we have observed, in the scale $j=4$, that subimages associated with $\theta=$ $\pm 15^{\circ}$ orientations appeared to hold more information about

Table $1 R$ parameter and the enhancement factors $\alpha$ for $E 1-E 4$

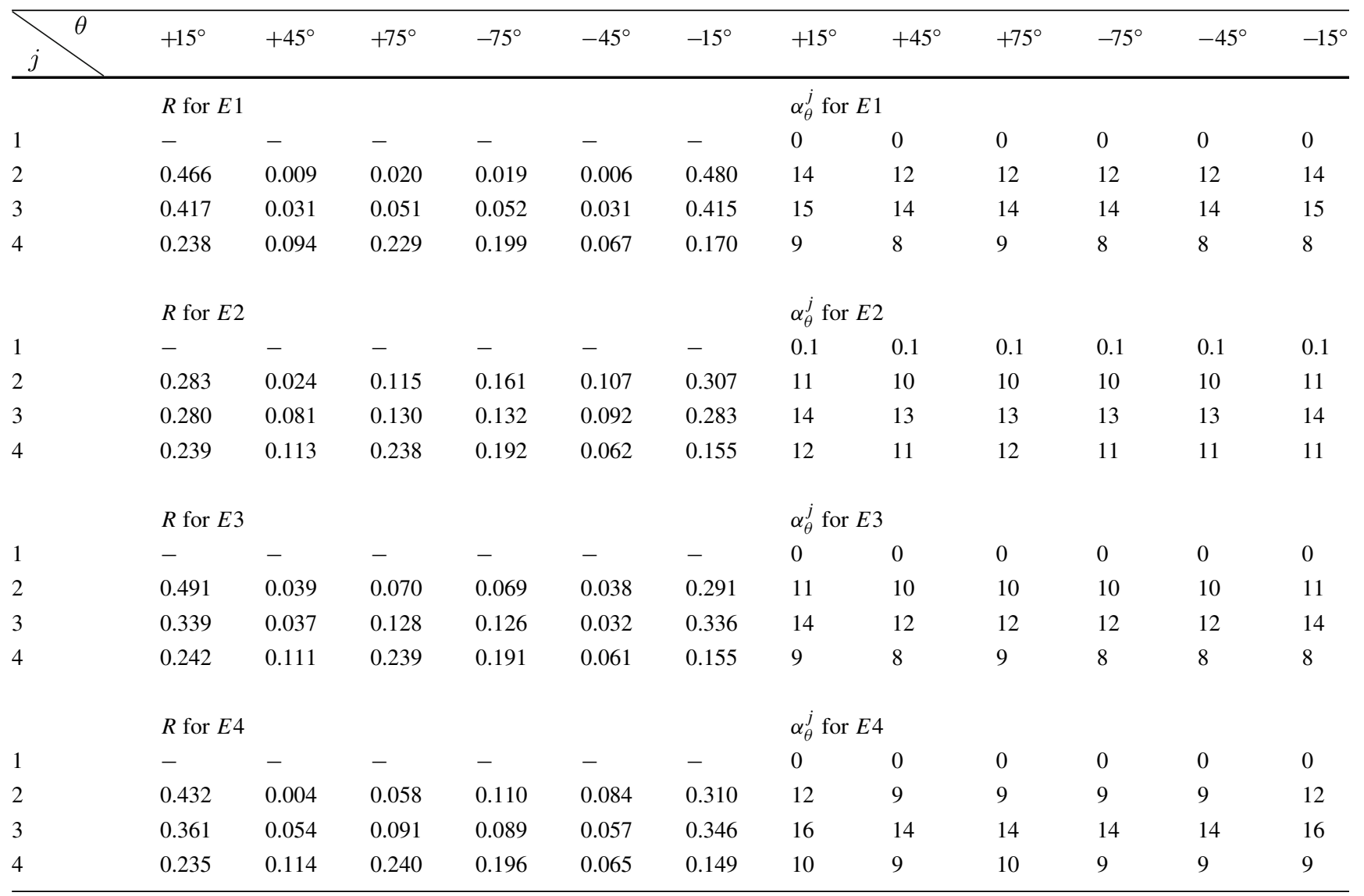


the plasma structure, this visual inspection was not entirely confirmed by the $R$ values calculated for these subimages. As shown in Table 1 , the larger $R$ values, which surpass the established threshold, correspond to $\theta=15^{\circ}$ and $75^{\circ}$ orientations. Thus, their wavelet coefficients undergo a higher enhancement factor: $\alpha_{\left\{15^{\circ}, 75^{\circ}\right\}}^{j=4}>\alpha_{\left\{-15^{\circ}, \pm 45^{\circ},-75^{\circ}\right\}}^{j=4}$. Hence, not only the visualization of the 2D DTCWT outcomes suffices to determine which wavelet coefficients should be enhanced, but the joint usage with the proposed $R$ parameter, have shown to play a fundamental role for providing an improvement of the plasma structures visualizations.

Analogous procedures were employed to enhance the wavelet coefficients on the subimages related to $E 2, E 3$, and $E 4$. Both the $R$ values and the enhancement factors $\alpha_{\theta}^{j}$ for these events are shown in Table 1. After the wavelet coefficient treatments, the images were reconstructed.

Images Reconstruction The 2D DTCWT was tested on the level 0.5 images which have no corrections for instrument response, stray light, etc. No background removal technique was used for the whole set of images analyzed in this work. The results provided by the wavelet analysis technique proposed are gathered in Fig. 3. In all images, an artificial occulter disk and a white annulus to represent the Sun's surface were added. At the left panels of Fig. 3 the original images representing Events $E 1-E 4$ are seen, whereas the reconstructed 2D DTCWT images are shown in the right panels. Next, each event is discussed.

E1: Eruptive Prominence on 02 June, 1998 This event is well known by the solar physics community. Its physical description can be found for instance in [20]. It was also used by [22] for validation of their wavelet technique.

Concerning the occurrence of artifacts by the image treatment, they can be identified as small dark points with white dots inside and some diffraction rings (which are also enhanced by the technique) around the periphery of the occulter disk.

In the reconstructed image (Fig. 3, E1, right panel), one can see that near the occulter disk there are some kinds of plasma threads (not seen in the original image) that appear to connect the outgoing structure with the solar photosphere. This characteristic indicates further that this structure is indeed a prominence. In addition, both its internal fine structure and twisted core are well resolved by this technique.

E2: CME on 23 November, 2000 According to [6], this event is referred to as a structured CME because, among other features, it reveals inherent fine structure discernible

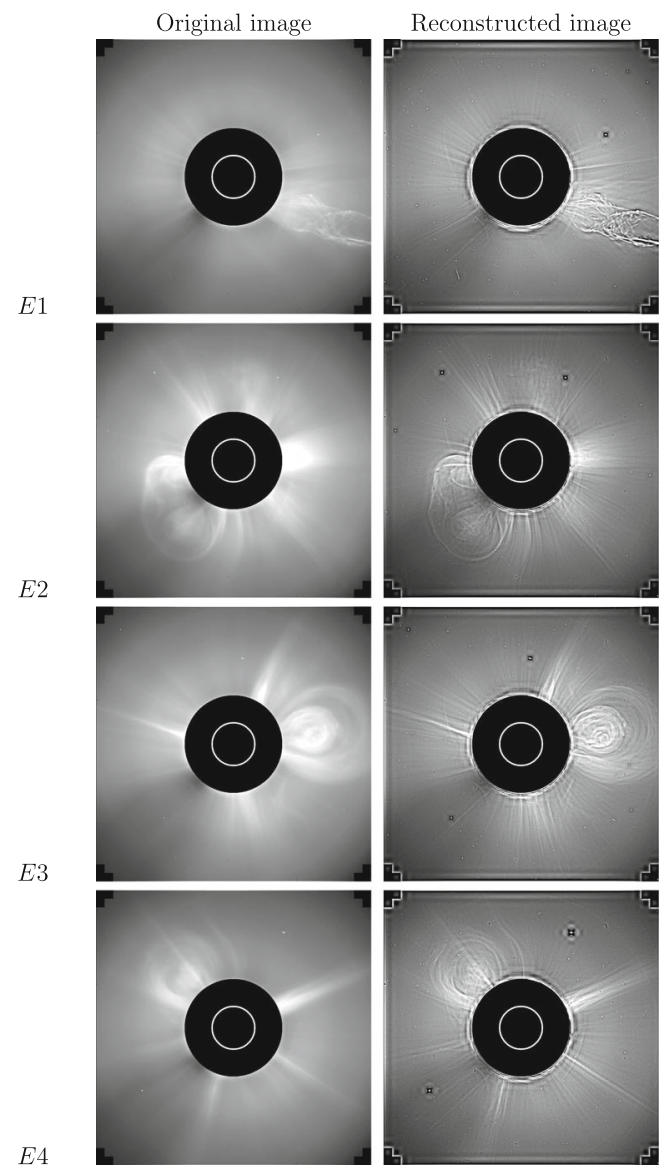

Fig. 3 The original images are at the left panel, and reconstructed 2D DTCWT images using the enhancement factors of Table 1 at the right panel. E1: Eruptive prominence on Jun. 02, 1998 at 13:31 UT, E2: CME on Nov. 23, 2000 at 13:31 UT, E3: CME on Oct. 06, 2001 at 08:06 UT, and E4: CME on Aug. 13, 2002 at 10:54 UT

from the ambient corona. This characteristic can be better visualized when our technique is applied (Fig. 3, E2, right panel). The edges become sharpened, which facilitates its tracking. In particular, the leading edge is readily discernable.

In contrast with the eruptive prominence shown in the previous section, this CME evolves mainly in the SouthEast quadrant of LASCO/C2 field-of-view, whereas the former plasma structure is in the South-West quadrant. It is shown that the 2D DTCWT can efficiently analyze features in the image independently of the structure's direction of propagation in the plane of sky.

E3: CME on 06 October, 2001 This CME evolves mainly in the North-West quadrant of LASCO/C2 field-of-view. It is also categorized as a structured CME according to the definition of [6]. In the reconstructed image (Fig. 3, E3, right panel), it is possible to see that the CME three-part 
structure, defined by [14] as a bright core within a dark lunette surrounded by a bright featureless loop, is better resolved by our technique. Its internal structure, although faint, is clearer than in the original image.

E4: CME on 02 August, 2002 The $E 4$ left panel in Fig. 3 shows this CME which evolves in the North-East quadrant of LASCO/C2 field-of-view. It is classified as a structured CME and presents the typical three-part structure. Their contours and fine structure are well resolved when our technique is applied. One can appreciate the sharpened edges appearing in the whole structure as well as in the North-East quadrant.

Our method was able to reduce the large level of noise contained in LASCO/C2 images. This could be achieved by means of a proper choice of the enhancement factors $\alpha_{\theta}^{j}$ particularly for the scale $j=1$.

\section{Conclusions}

In all treated images, highlighting and sharpening of edges as well as enhancement of CMEs bright cores visualizations were obtained. These results help solar physicists, for instance, in an unambiguous identification of CMEs three-part structures. The determination of plasma-structure kinematic properties can also be facilitated by the employed technique. As a natural consequence, morphological studies can be carried out more easily.

In this work, our wavelet methodology has shown the ability to identify in a precise way diffuse coronal structures by enhancing both their boundaries and internal features based on multidirectional analysis. For future work, exploratory analysis to deal with different angle orientations can be done with 2-D continuous wavelet transform [1], for instance. However, in this case, the computational cost is much larger than the DT CWT, and the complexity of the analysis and the redundancy of the data are greatly enhanced.

Much of the DTCWT's potential can still be exploited due to the inherent multiscale nature of solar images. We have shown that even with the limitation of no usage of both pre-processing and background removal techniques, a great improvement in the visualizations of the plasma structures in LASCO/C2 images was achieved. Therefore, our method can be improved by applying models to get rid of undesirable background and side effects.

Acknowledgments The authors thank the grants provided by FAPESP 2014/ 21229-9, 2007/ 07723-7; CAPES 126/2012-83; CNPq 307511/2010-3, 312246/2013-7; the authors also thank ESA and NASA for providing the $\mathrm{SOHO} / \mathrm{LASCO}$ images used in this work.
$\mathrm{SOHO}$ is a project of international collaboration between the European Space Agency (ESA) and NASA. The authors are grateful to Eng. Varlei Menconi by the computational assistance (FAPESP 2008/09736-1, CNPq 302451/2013-7), and Prof. Dr. Nick Kingsbury for the fruitful scientific discussions, the codes, and the lectures given in the Workshop in Wavelet Applications (WWLET-2012).

\section{References}

1. J.-P. Antoine, R. Murenzi, P. Vandergheynst, S.T. Ali, Two dimensional wavelets and their relatives (Cambridge, 2008)

2. G.E. Brueckner, R.A. Howard, M.J. Koomen, C.M. Korendyke, D.J. Michels, J.D. Moses, D.G. Socker, K.P. Dere, P.L. Lamy, A. Llebaria, M.V. Bout, R. Schwenn, G.M. Simnett, D.K. Bedford, C.J. Eyles, The large angle spectroscopic coronagraph (LASCO). Sol. Phys. 162, 357-402 (1995)

3. L. Burlaga, E. Sittler, F. Mariani, R. Schwenn, Magnetic loop behind an interplanetary shock: Voyager, Helios, and IMP 8 observations. J. Geophys. Res. 86(A8), 6673-6684 (1981)

4. L.F. Burlaga, K.W. Behannon, L.W. Klein, Compound streams, magnetic clouds, and major geomagnetic storms. J. Geophys. Res. 92(A6), 5725-5734 (1987)

5. J.P. Byrne, P.T. Gallagher, R.T.J. McAteer, C.A. Young, The kinematics of coronal mass ejections using multiscale methods. A\&A 495(1), 325-334 (2009)

6. H. Cremades, V. Bothmer, On the three-dimensional configuration of coronal mass ejections. A\&A 422, 307-322 (2004)

7. H. Cremades, O.C. St. Cyr, G. Stenborg, Revealing the inside of coronal mass ejections. in SOHO-17. 10 Years of SOHO and Beyond, 617, ESA, (2006)

8. I. Daubechies, Ten Lectures on Wavelets. 61 of CBMS-NSF Regional Conference Series in Applied Mathematics (SIAM, Philadelphia, 1992)

9. K.P. Dere, G.E. Brueckner, R.A. Howard, D.J. Michels, J.P. Delaboudiniere, LASCO and EIT observations of helical structure in coronal mass ejections. ApJ 516(1), 465 (1999)

10. E. Echer, W.D. Gonzalez, M.V. Alves, On the geomagnetic effects of solar wind interplanetary magnetic structures. Space Weather 4(6) (2006)

11. J.T. Gosling, E. Hildner, R.M. MacQueen, R.H. Munro, A.I. Poland, C.L. Ross, Mass ejections from the sun: a view from Skylab. J. Geophys. Res. 79(31), 4581-4587 (1974)

12. J.C. Goswami, A.K. Chan, Fundamentals of Wavelets: Theory, Algorithms, and Applications, $2^{\text {nd }} \mathrm{Ed}$ (Wiley, 2011)

13. T.A. Howard, D. Nandy, A.C. Koepke, Kinematic properties of solar coronal mass ejections: correction for projection effects in spacecraft coronagraph measurements. J. Geophys. Res. 113(A1), A01104 (2008)

14. R.M.E. Illing, A.J. Hundhausen, Observation of a coronal transient from 1.2 to 6 solar radii. J. Geophys. Res. 90(A1), 275-282 (1985)

15. N.G. Kingsbury, The dual-tree complex wavelet transform: A new efficient tool for image restoration and enhancement. in European Signal Processing Conference, EUSIPCO 98. Rhodes, USA., pp. 319-322, (1998a)

16. N.G. Kingsbury, The dual-tree complex wavelet transform: A new technique for shift invariance and directional filters. in IEEE Digital Signal Processing Workshop DSP. Bryce Canyon, USA., pp. 319-322, (1998b)

17. N.G. Kingsbury, Complex wavelets for shift invariant analysis and filtering of signals. Appl. Comput. Harmon. A 10(3), 234-253 (2001) 
18. S. Mallat, A Wavelet Tour of Signal Processing, (Wavelet Analysis \& Its Applications), $2^{\text {nd }}$ Ed. (Academic Press, 1999)

19. H. Morgan, S. Habbal, R. Woo, The depiction of coronal structure in white-light images. Sol. Phys. 236, 263-272 (2006)

20. S.P. Plunkett, A. Vourlidas, S. Šimberová, M. Karlický, P. Kotrč, P. Heinzel, Y.A. Kupryakov, W.P. Guo, S.T. Wu, Simultaneous SOHO and ground-based observations of a large eruptive prominence and coronal mass ejection. Sol. Phys. 194, 371-391 (2000)

21. I.W. Selesnick, R.G. Baraniuk, N.G. Kingsbury, The dual-tree complex wavelet transform. IEEE Signal Process. Mag. 22(6), 123-151 (2005)
22. G. Stenborg, P.J. Cobelli, A wavelet packets equalization technique to reveal the multiple spatial-scale nature of coronal structures. A\&A 398(3), 1185-1193 (2003)

23. G. Stenborg, A. Vourlidas, R.A. Howard, A fresh view of the extreme-ultraviolet corona from the application of a new imageprocessing technique. ApJ 674, 1201-1206 (2008)

24. D. Tripathi, G. Stenborg, On the internal structures of coronal mass ejections. in The Dynamic Sun: Challenges for Theory and Observations. 600, ESA, (2005)

25. J. Zhang, K.P. Dere, R.A. Howard, M.R. Kundu, S.M. White, On the temporal relationship between coronal mass ejections and flares. ApJ 559, 452-462 (2001) 